\title{
ABILSEN: Uma Abordagem para Inclusão do Licenciado em Computação no Ensino Básico
}

\author{
Sebastião Rogério da Silva Neto ${ }^{1}$, Higor Ricardo M. Santos ${ }^{1}$, Wilk Oliveira dos \\ Santos ${ }^{1}$ \\ ${ }^{1}$ Universidade de Pernambuco (UPE) \\ Caixa Postal 55.294-902 - Garanhuns - PE - Brasil \\ \{sebast.rogers@gmail.com, higor.monteiro@upe.br, \\ wilk.upe@gmail.com\}
}

\begin{abstract}
The use of ICT (Information and Communication Technologies and Computer Science education in schools has fostered discussions about new practices and methods to assist in the process of teaching and learning. The degree courses in Computer Science empower professionals to develop educational software, applying ICT in Education and teaching in basic Computation, technical and professional levels. However, it still does not exist in Brazil to the discipline in the basic education curriculum. This research presents an approach to the inclusion of a degree in Computation structured and integrated way to the disciplines of the school curriculum. The results of the empirical study show a high satisfaction of the approach both students and the discipline teacher.
\end{abstract}

Resumo. A utilização das Tecnologias de Informação e Comunicação - (TICs) $e$ o ensino de Computação nos espaços escolares têm fomentado discussões sobre novas práticas e métodos para auxiliar no processo de ensino e aprendizagem. Os cursos de Licenciatura em Computação formam profissionais para desenvolver softwares educativos, aplicar TICs na Educação e ensinar Computação nos níveis básico, técnico e profissionalizante. Entretanto, ainda não existe no Brasil a disciplina de Computação no currículo da educação básica. Esta pesquisa apresenta uma abordagem para a inclusão do Licenciado em Computação de forma estruturada e integrada às disciplinas do currículo escolar. Os resultados do estudo empírico demonstram uma alta satisfação da abordagem tanto dos alunos quanto do professor da disciplina.

\section{Introdução}

A educação escolar, em todos os níveis, sempre esteve restringida a lugares e tempos determinados, tais como, salas de aula, calendário escolar, grade curricular e modelos pedagógicos centrados no professor. Esses fatores procuram ajudar o modo como cada indivíduo consegue avançar no processo de aprendizagem. No entanto, existem os limites do conteúdo programático, do tempo de aula, das normas legais e, por conta disso, boa parte dos professores se tornam previsíveis, não surpreende os alunos e repete fórmulas e sínteses (MORAN, 2003).

A integração das Tecnologias Digitais da Informação e Comunicação (TDIC's) dentro de espaços escolares tem fomentado discussões sobre novas práticas e métodos 
no processo de ensino e aprendizagem. O Model Curriculum K-12 Computer Science ${ }^{1}$ por exemplo, destaca que a grande maioria das profissões do século XXI exige uma compreensão da Ciência da Computação. Profissionais de arte, entretenimento, comunicação, saúde, entre outros, precisam ter conhecimentos da Computação, enquanto ciência, na busca por soluções de problemas de áreas diversas, bem como na construção dessas soluções (FRANÇA et al. 2012). Com isso, além da integração dessas tecnologias (computadores, celulares, tablets, etc.), torna-se relevante para os alunos das séries iniciais, o ensino de conceitos fundamentais da Ciência da Computação. Esses conceitos propiciam o desenvolvimento de habilidades que são exigidas no mundo atual, tais como, criatividade e raciocínio lógico. Habilidades estas que auxiliam na resolução de qualquer tipo de problema.

A finalidade do uso de tecnologia na educação é muito bem definida por proporcionar vivências intensas de colaboração. Essa dinâmica requer o rearranjo das formas de ação e interação facilitadas pelas tecnologias digitais. Além disso, ela demanda um entendimento que supere os limites de uma simples metodologia para fomentar novos perfis de professores e alunos, na busca por formas de conceber o ensino e a aprendizagem em sintonia com as estruturas cognitivas mobilizadas em função do contexto cultural, político, social e econômico do século XXI (SHNEIDERMAN, 2006). Diante dessa necessidade, o curso de Licenciatura em Computação foi construído com a intenção de formar profissionais com a capacidade de desenvolver (programar) e a aplicar tecnologias para auxiliar a educação, como também ensinar Ciência da Computação na educação básica, técnica e profissionalizante. No entanto, atualmente não existe um espaço para esses profissionais atuarem na educação básica, pois elas ainda não oferecem um currículo onde ele possa lecionar Computação como uma disciplina.

Tendo em vista a relevância do que foi exposto pela Sociedade Brasileira de Computação (SBC), a qual defende que o ensino de Computação se inicie desde o ensino fundamental, a exemplo de outras ciências, como Física, Matemática, Química, Biologia, é primordial a introdução de conceitos de Ciência da Computação na Educação Básica como forma de aprimorar o raciocínio lógico e computacional das crianças, pelo seu caráter transversal a todas as ciências (NUNES, 2011). Nesse contexto, esta pesquisa se propôs a investigar o seguinte problema: como incluir o Licenciado em Computação na educação básica brasileira, sabendo-se que não existe um currículo oficial que especifique tal inclusão?

\section{Revisão da Literatura}

Para o desenvolvimento desta pesquisa, utilizamos como base as referidas teorias: Pensamento Computacional, Jogos Educativos e Objetos de Aprendizagem. A seguir, apresenta-se uma descrição sucinta de cada uma.

\footnotetext{
${ }^{1}$ Model Curriculum K-12 Computer Science: Report of the ACM K-12 Task Force Computer Science Curriculum Committee, $2011 . \quad$ Disponível em: http://www.csta.acm.org/Curriculum/sub/CurrFiles/CSTA_K-12_CSS.pdf
} 


\subsection{Pensamento Computacional}

O Pensamento Computacional baseia-se em fundamentos da Computação, envolvendo a resolução de problemas, a capacidade de projetar sistemas e a compreensão do comportamento humano (WING, 2008). É um pensamento analítico que compartilha com a Matemática a resolução de problemas, com a Engenharia a concepção e avaliação de um sistema grande e complexo que opera dentro dos limites do mundo real e com a Ciência a compreensão sobre computabilidade, inteligência, a mente e o comportamento humano (WING, 2008).

O Pensamento Computacional constitui uma habilidade fundamental, não apenas para os cientistas da Computação, influenciando a pesquisa em várias áreas do conhecimento (BUNDY, 2007). A introdução do pensamento computacional na educação básica provê os recursos cognitivos necessários à resolução de problemas, transversal a todas as áreas do conhecimento (NUNES, 2011).

\subsection{Jogos Eletrônicos Educativos}

De acordo com Sá et al. (2007), a utilização de jogos eletrônicos nas atividades de ensino proporciona ao aprendiz momentos lúdicos e interativos como etapas do processo de aprendizagem. Os jogos têm a capacidade de auxiliar o professor na apresentação de conceitos de determinados assuntos, desde que sejam desenvolvidos e trabalhados de forma crítica, possibilitando a aprendizagem significativa do aluno (GROS, 2007).

Para que os jogos possam ser caracterizados como educativos, eles "devem possuir objetivos pedagógicos e sua utilização deve estar inserida em um contexto e em uma situação de ensino baseados em uma metodologia que oriente o processo, através da interação, da motivação e da descoberta, facilitando a aprendizagem de um conteúdo" (Prieto et al. 2005, p. 10).

\subsection{Objetos de Aprendizagem}

Conforme o Comitê de Padronização das Tecnologias de Aprendizagem (LTSC), um Objeto de Aprendizagem (OA) é definido como "qualquer entidade digital ou não que pode ser utilizada, reutilizada ou referenciada durante o aprendizado apoiado pela tecnologia." (IEEE, 2002).

Segundo SINGH apud BETTIO E MARTINS (2001), um OA para ser bem estruturado deve possuir os seguintes elementos: 1) Objetivos: tem como intenção demonstrar ao aprendiz o que ele poderá aprender a partir do estudo desse OA. Também poderá conter uma lista dos conhecimentos prévios necessários para um bom aproveitamento de todo o conteúdo disponível; 2) Conteúdo instrucional: deverá ser apresentado todo o material didático necessário para que no término, o aluno possa alcançar os objetivos citados no item anterior; 3) Prática e FeedBack: Uma das características importantes dos OA's é que a cada final de utilização julga-se necessário que o aprendiz verifique se o seu desempenho atingiu as expectativas, caso contrário, o aprendiz deve ter a liberdade de reutilizar o objeto quantas vezes julgar necessário.

Para serem inseridos em um ambiente de aprendizagem, é importante ainda que os OA's apresentem as seguintes características: Reusabilidade: reutilizável diversas vezes em diversos ambientes de aprendizagem; Adaptabilidade: adaptável a qualquer ambiente de ensino; Granularidade: conteúdo em componentes, para facilitar sua reusabilidade; Acessibilidade: acessível facilmente via Internet para ser usado em 
diversos locais; Durabilidade: possibilidade de continuar a ser usado, independente da mudança de tecnologia; e Interoperabilidade: habilidade de operar através de uma variedade de hardware, sistemas operacionais e intercâmbio entre os sistemas.

\subsection{Trabalhos Relacionados}

Na literatura é possível encontrar diversos trabalhos que apresentam experiências sobre a aplicação do ensino de Computação na educação básica no Brasil, tais como, (COSTA ET AL. 2012; SCAICO, 2012; FRANÇA ET AL. 2012). No entanto, não foi evidenciado na revisão da literatura trabalhos que propõem abordagens para inclusão do Licenciado em Computação.

\section{Metodologia}

Esta pesquisa se configura como empírica de natureza qualitativa. Segundo Bhattacharya (2008), uma pesquisa empírica tem como objetivo principal observar um fenômeno no mundo social e então gerar conhecimento sobre este fenômeno. Pesquisa empírica é baseada em ideais de credibilidade, confiabilidade e outros princípios de rigor fundamentais, os quais devem estar interligados e envolvidos com os objetivos e o problema da pesquisa (PATTON, 2002). A Figura 1 apresenta a metodologia utilizada nesta pesquisa.

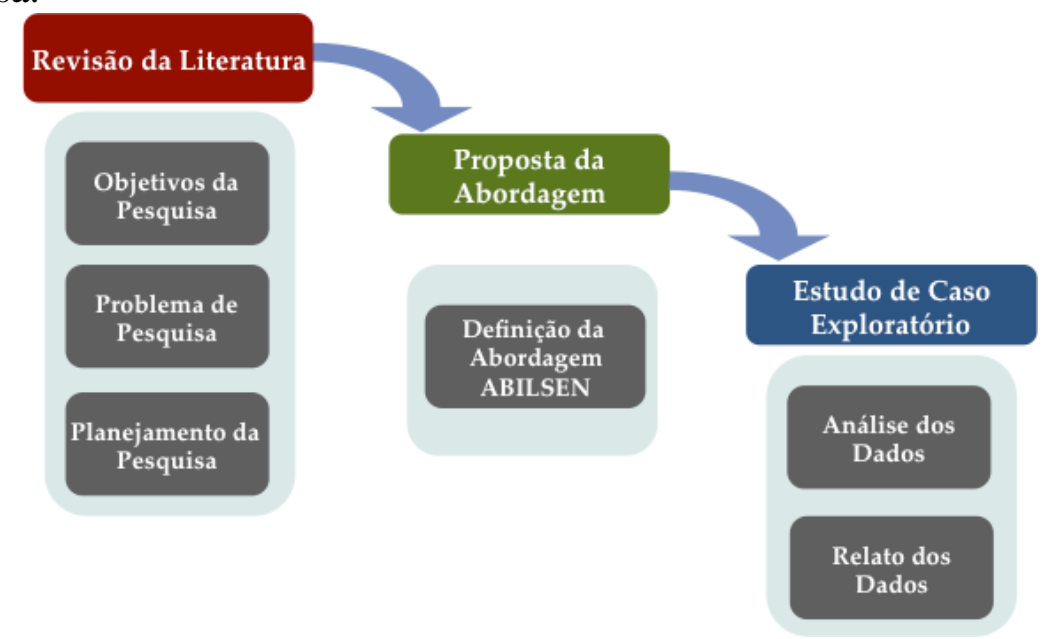

Figura 1. Metodologia da Pesquisa

O estudo empírico foi realizado através de um estudo de caso exploratório. Um ponto importante no planejamento de um estudo de caso é a definição do caso e da unidade de análise. $\mathrm{O}$ caso pode ser um indivíduo, um grupo de pessoas, um evento ou uma entidade (YIN, 2001). Da mesma forma, o projeto, indivíduo, grupo, pode também constituir a unidade de análise do estudo de caso (Runeson \& Höst, 2008). Yin (2001) orienta que a definição do caso e da unidade de análise está relacionada à forma como foram definidas as questões de pesquisa. Desse modo, o caso investigado é a escola selecionada para participar desta pesquisa. E a unidade de análise é o ensino de Computação atrelado as demais disciplinas do currículo escolar.

\section{Resultados}

\subsection{Proposta da Abordagem ABILSEN}

Com a intenção de investigar como incluir o Licenciado em Computação no ensino básico, a abordagem proposta nesta pesquisa teve como base as etapas do método 
interativo de gestão PDCA (Plan - Do - Check - Act). Segundo LIMA (2006), o Ciclo PDCA (Planejar, Executar, Verificar e Refinar) é uma ferramenta utilizada para a aplicação das ações de controle dos processos, tal como estabelecimento da "diretriz de controle", planejamento da qualidade, manutenção de padrões e alteração da diretriz de controle, ou seja, realizar melhorias.

Com inspiração nesse método, foi definida a Abordagem de Inclusão do Licenciado em Computação no Ensino Básico - ABILSEN. Por não existir um currículo de Computação na educação básica, esta abordagem possibilitará o Licenciado em Computação atuar em conjunto com os professores das demais disciplinas, tais como, Matemática, Geografia e Física. O ensino de Computação através da ABILSEN acontecerá transversalmente às outras ciências que já estão inseridas no currículo escolar. A Figura 2 apresenta uma adaptação do ciclo PDCA de acordo com a abordagem desenvolvida nesta pesquisa. Diante disso, foram definidas etapas específicas para cada uma das fases do ciclo. A seguir, as etapas definidas na ABILSEN são descritas sucintamente de acordo com sua importância e as atividades que o Licenciado em Computação deverá desempenhar ao longo de uma disciplina escolar.

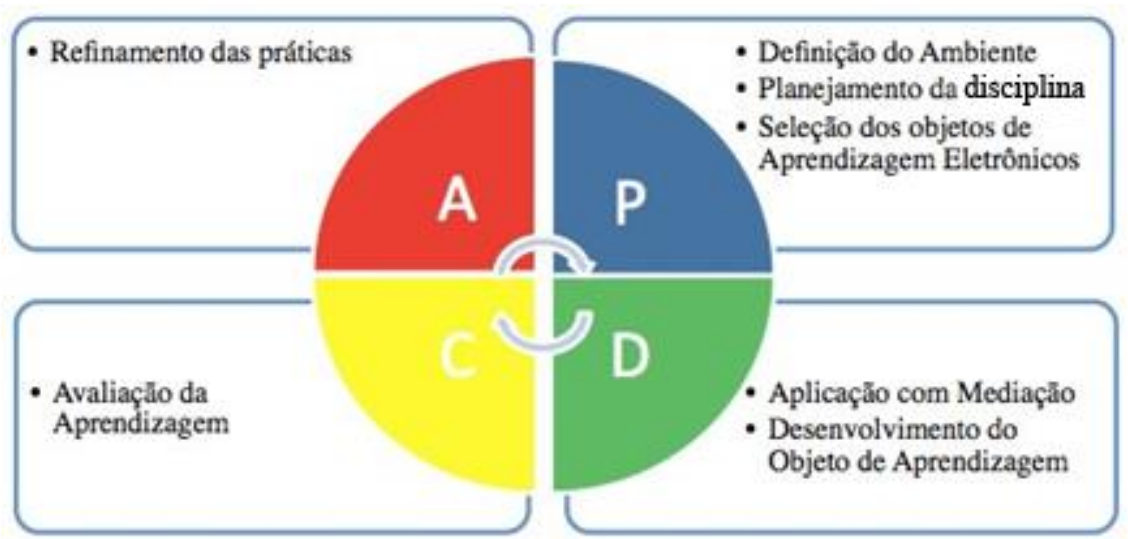

Figura 2. Abordagem ABILSEN

Definição do Ambiente: permite ao Licenciado em Computação uma visão ampla do ambiente em que será aplicada a abordagem. Nessa etapa, o Licenciado em Computação verificará os recursos tecnológicos (Computadores, Internet e softwares) disponíveis na escola, como também apresentará a abordagem ao corpo docente e a gestão da escola.

Planejamento da Disciplina: o professor da disciplina selecionada e o Licenciado em Computação devem se reunir para planejar e organizar os assuntos que serão trabalhados por ambos. Com isso, além dos conhecimentos prévios do professor da disciplina, será colocado em pauta também a sua experiência prévia e a dificuldade dos alunos na disciplina em questão.

Seleção de Objetos de Aprendizagem Eletrônicos: De acordo com Nunes (2004), "Os OA's quando bem escolhidos ajudam o aluno em várias etapas do processo de aprendizagem, tais como, relacionar novos conhecimentos com os que já sabiam fazer, testar hipóteses, pensar onde aplicar o que estão aprendendo, expressar-se por meio de várias linguagens, aprender novos métodos, conceitos, além de motivar e contextualizar um novo conteúdo curricular a ser tratado". Nessa etapa, o Licenciado deve pesquisar OA's que obedeçam as características citadas acima, analisar o OA 
relacionando-o ao assunto selecionado na fase de planejamento, testar os OA's com antecedência, instalar nas máquinas da escola, apresentar e discutir com o professor da disciplina a respeito do OA escolhido e como será abordado ao longo das aulas.

Aplicação com Mediação: Nessa etapa, o Licenciado em Computação conduzirá os alunos ao laboratório de informática para aplicar os OA's selecionados com o professor da disciplina. O Licenciado junto com o professor da disciplina deverão desenvolver atividades que possam utilizar esses artefatos com os alunos no laboratório.

Desenvolvimento do Objeto de Aprendizagem: A fim de qualificar o OA, propõe-se a adaptação do modelo de processo de construção utilizado pela Rede Internacional Virtual de Educação - RIVED ${ }^{2}$, de forma a caracterizar a participação dos alunos e professores, e não dos especialistas. Esta adaptação contempla a participação de alunos em todas as etapas do processo de desenvolvimento e é flexível, pois permite ao aluno voltar a etapas anteriores para atualizar e refazer o processo de construção do OA. Nessa etapa, o Licenciado junto com os alunos irão desenvolver (programar) um OA, a fim de pôr em prática conceitos da computação.

Avaliação da Aprendizagem - Modelo Kirkpatrick: Nessa etapa, o Licenciado pode avaliar a eficácia da abordagem através do modelo de avaliação de treinamentos de Kirkpatrick (1994), o qual é dividido em quatro níveis. Cada nível tem sua importância e, conforme se passa de um nível para outro sequente, o processo se torna mais complexo e demorado, porém fornece resultados mais valiosos. Os níveis são: (1) Reação: onde se mede a satisfação e valor percebido do treinamento pelos participantes; (2) Aprendizagem: identifica o quanto os participantes podem mudar de atitude, ampliar seus conhecimentos e/ou habilidades; (3) Comportamento: identifica o quanto os participantes mudaram seu comportamento em decorrência do que foi aprendido; (4) Resultados: identifica os ganhos obtidos no treinamento. O presente modelo será utilizado a fim de levantar os dados referentes a efetividade da abordagem.

Refinamento das Práticas: Nesta etapa, o Licenciado em Computação analisará de forma crítica todas as suas atividades de acordo com a eficiência e a eficácia da abordagem. Nessa análise, será levado em consideração os pontos positivos, negativos e lições aprendidas com a intenção de melhorar continuamente a aplicação da ABILSEN. Essa análise também será realizada em conjunto com o professor da disciplina selecionada. É preciso verificar se a abordagem obteve êxito em todas as etapas, ele deve então buscar novos OA's junto ao professor da disciplina, buscando manter a mesma metodologia utilizada anteriormente, bem como aplicar essa mesma abordagem com a adição destes novos objetos em diferentes turmas.

\subsection{Estudo de Caso Exploratório}

Com a intenção de verificar a aderência da abordagem no ambiente prático, foi realizado um estudo de caso exploratório em uma escola situada na região Agreste do estado e Pernambuco. Escola de Referência em Ensino Médio Augusta Cordeiro de Melo na turma do $8^{\circ}$ Ano $7^{a}$ série. Esta escola localiza-se na cidade de Calçado - PE. O período de realização deste estudo foi no período da tarde, contando com a participação de 20 alunos, a aplicação da abordagem aconteceu no laboratório de informática e na sala de

\footnotetext{
${ }^{2}$ Site do RIVED: http://rived.mec.gov.br/projeto.php
} 
aula com a utilização de data show e apresentação de slides para explanação do conteúdo. O estudo empírico realizado de acordo com a abordagem anteriormente descrita é apresentado a seguir.

Definição do Ambiente: Nesta etapa, foi desenvolvida uma carta de apresentação e pedido de permissão para a diretora da escola onde foi aplicada a abordagem ABILSEN. Após a sua permissão, foram verificados os recursos tecnológicos da escola, onde se constatou que os mesmos encontravam-se em bom estado de conservação. Na sequência, foi apresentada a abordagem ao corpo docente da escola, que por sua vez se prontificou a colaborar com o mesmo. Nesse sentido, atendendo a um pedido da direção da escola, o projeto foi aplicado na disciplina de Matemática em função dos estudantes da escola apresentarem grande dificuldade nesta disciplina, bem como a mesma ter um nível alto de reprovação de acordo com a coordenação da escola.

Planejamento da Disciplina: Nesta etapa, aconteceram três reuniões com o professor da disciplina de Matemática, que explanou suas dificuldades no ensino de conteúdo, além de mostrar ao grupo proponente da abordagem (autores desta pesquisa) a ementa que devia ser abordada na disciplina.

Seleção de Objetos de Aprendizagem Eletrônicos: Nesta etapa, foram pesquisados na internet por softwares que pudessem apresentar de forma lúdica os assuntos apresentados pelo professor de matemática. Os softwares escolhidos, bem como suas características são apresentados no Tabela 1.

Tabela 1. Objetos de Aprendizagem Selecionados

\begin{tabular}{|c|l|}
\hline Nome & \multicolumn{1}{|c|}{ Descrição } \\
\hline Enigma das Frações & $\begin{array}{l}\text { Este software é jogo 2D que tem por objetivo apresentar diferentes conceitos de } \\
\text { fração. É uma excelente atividade para reforçar o assunto de divisões por } \\
\text { frações de forma lúdica. }\end{array}$ \\
\hline Mundo de Euclides & $\begin{array}{l}\text { Este software é um jogo 2D para o ensino fundamental II com o intuito de } \\
\text { trabalhar com apresentação e análise de conceitos iniciais da geometria espacial } \\
\text { (Pontos, Retas, Segmentos e Planos). }\end{array}$ \\
\hline Feche a Caixa & $\begin{array}{l}\text { Este software é um jogo secular que tem por objetivo fechar o maior número de } \\
\text { caixas, perdendo o mínimo de pontos. É uma excelente atividade para estimular } \\
\text { o cálculo mental. }\end{array}$ \\
\hline Ludo Educativo & $\begin{array}{l}\text { Este software é um jogo 2D no estilo tabuleiro, porém com fins educativos. } \\
\text { Nele, você deve responder as perguntas de forma correta para vencer. }\end{array}$ \\
\hline Mangahigh & $\begin{array}{l}\text { É um recurso matemático adaptativo em jogos concebidos para o ensino } \\
\text { híbrido. Nele, estão disponíveis diversos jogos para alunos do ensino } \\
\text { fundamental I e II trabalharem apenas assuntos relacionados a matemática. }\end{array}$ \\
\hline
\end{tabular}

Aplicação com Mediação: Nesta etapa, a equipe proponente da abordagem, com a mediação do professor da disciplina de Matemática, reuniram-se com os estudantes no laboratório de informática da escola para a condução das aulas com os objetos de aprendizagem selecionados. A Figura 3 demonstra os alunos interagindo na aplicação dos OA's. 


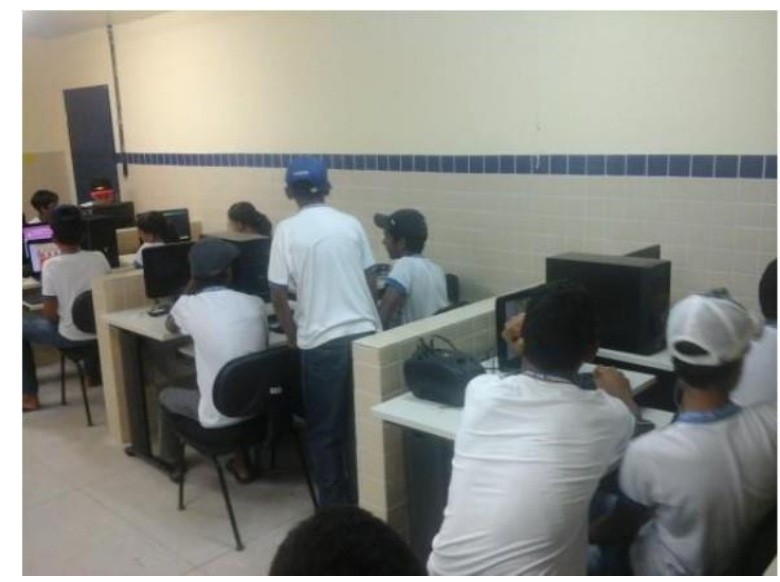

Figura 3. Aplicação dos OA's

Desenvolvimento do Objeto de Aprendizagem: Na presente etapa os alunos foram divididos em 3 grandes grupos. Na sequência, o Licenciado em Computação apresentou em forma de slides os conceitos básicos de Computação (algoritmo, programa, variável, condicionais). É importante ressaltar que a divisão dos alunos em grupos aconteceu devido o sistema operacional do laboratório, o qual não permitiu a instalação do ambiente GameMaker 8.0. Com isso, os pesquisadores ofereceram três notebooks para sanar esse problema. Em seguida, os alunos foram apresentados ao GameMaker 8.0 e suas funcionalidades para criação dos OA's.

Com isso, os alunos foram convidados a desenvolver um jogo na plataforma supracitada, que permitiu aos mesmos colocar em prática os conceitos de Matemática e Computação anteriormente mencionados, de uma forma lúdica, por meio de linguagem de programação drop-and-down oferecida pela plataforma. A dinâmica dessa etapa aconteceu no período de duas horas e foram desenvolvidos os jogos pelas três equipes, sob a supervisão dos proponentes da abordagem e pelo professor de matemática.

Avaliação da Aprendizagem: A avaliação apresentada nesta seção segue o modelo de Kirkpatrick (1994), sendo abordados aspectos relacionados a: Atenção, Relevância, Confiança, Satisfação, Desafio, Habilidade, Divertimento e Conhecimento. Nesse sentido, ao final da aplicação dos OA's foi apresentado um questionário estruturado, baseado na escala Likert de 5 pontos, com 8 questões para cada estudante. As questões continham os seguintes pontos: Discordo Fortemente (DF), Discordo (D), Indeciso (I), Concordo (C), Concordo Fortemente (CF). A seguir são apresentados os resultados obtidos nesta etapa da abordagem.

A Tabela 2 apresenta os resultados obtidos na etapa de Avaliação da Aprendizagem. Ao analisar, pode-se perceber que $80 \%$ dos alunos concordam que os softwares retêm a sua atenção, bem como $90 \%$ deles consideram os objetos de aprendizagem relevantes. É interessante perceber ainda que toda a turma ficou satisfeita com a experiência. Assim, os resultados obtidos nesta etapa da avaliação oportunizaram perceber que os objetos de aprendizagem selecionados apresentam seus conteúdos de maneira lúdica, tornando agradável o ato de jogar e, por conseguinte, corroborando para que o mesmo pudesse permanecer por mais tempo no jogo. 
Tabela 2. Resultados da Avaliação do Questionário

\begin{tabular}{|l|c|c|c|c|c|}
\cline { 2 - 6 } \multicolumn{1}{c|}{} & DF & D & I & C & CF \\
\hline Atenção & $0 \%$ & $5 \%$ & $15 \%$ & $50 \%$ & $30 \%$ \\
\hline Relevância & $0 \%$ & $0 \%$ & $10 \%$ & $40 \%$ & $50 \%$ \\
\hline Confiança & $0 \%$ & $40 \%$ & $10 \%$ & $10 \%$ & $40 \%$ \\
\hline Satisfação & $0 \%$ & $0 \%$ & $0 \%$ & $60 \%$ & $40 \%$ \\
\hline Desafio & $0 \%$ & $0 \%$ & $30 \%$ & $50 \%$ & $20 \%$ \\
\hline Habilidade & $10 \%$ & $10 \%$ & $0 \%$ & $80 \%$ & $0 \%$ \\
\hline Divertimento & $0 \%$ & $0 \%$ & $40 \%$ & $20 \%$ & $40 \%$ \\
\hline Conhecimento & $10 \%$ & $20 \%$ & $10 \%$ & $40 \%$ & $20 \%$ \\
\hline
\end{tabular}

Refinamento: Nesta etapa, os pesquisadores analisaram de forma crítica todas as suas atividades de acordo com a eficiência e a eficácia da abordagem. Nessa análise, foi levado em consideração os pontos positivos, negativos e lições aprendidas. A análise também foi realizada em conjunto com o professor da disciplina de Matemática. Apesar de alguns entraves devido ao sistema operacional do laboratório, observou-se que a abordagem obteve êxito em todas as etapas. Com a análise devidamente realizada, foi gerado um relatório com todas as modificações e melhorias a serem realizadas.

\section{Considerações Finais}

Diante da necessidade de fomentar a criatividade, raciocínio lógico e abrir espaço de mercado para os formados em Licenciatura em Computação, esta pesquisa teve como principal contribuição a proposição e aplicação de uma abordagem, denominada de ABILSEN, para incluir os Licenciados em Computação na educação básica através do estímulo do Pensamento Computacional. Essa abordagem foi desenvolvida baseada no ciclo PDCA, o qual tem como fases o Planejamento, Aplicação, Avaliação e Refinamento.

O trabalho foi iniciado através de uma pesquisa bibliográfica, resultando em um conjunto de conceitos de Computação, ambientes de desenvolvimento e OA's. Os quais foram utilizados no desenvolvimento e aplicação da abordagem. Com a pesquisa realizada, foi detectado que não há um currículo para Computação e nem alternativas para que o Licenciado em Computação atue de maneira efetiva no ensino básico.

Observou-se que o professor de Matemática, que atuou em conjunto com os pesquisadores, ficou satisfeito com a iniciativa e com as possibilidades que as TIC's podem trazer para a sala de aula. Após o término, outros professores da escola demonstraram interesse em aplicar a ABILSEN em suas disciplinas. De modo geral, a escola em si reconheceu a importância do ensino da Computação, enquanto Ciência, e apoiaram a inclusão do Licenciado em Computação no ambiente escolar. Ressalta-se que devido ao limite de páginas, foram omitidas fotos da experiência e relatos dos participantes.

Por fim, sugere-se como trabalhos futuros a replicação desta abordagem em mais escolas e com outras disciplinas para comparar os resultados com esta. Além disso, seria interessante a utilização de outras plataformas para desenvolvimento de OA's, tais como, os softwares Scratch, Robomind e RPG Maker. 


\section{Referências}

Bhattacharya, H. (2008) "Empirical Research". In: GIVEN, L. M. The SAGE Encyclopedia of Qualitative Research Methods. 1. ed. California: Sage Publications, v. 1 e 2. Cap. Entrada E, p. 253-255.

Bundy, A. (2007) "Computational thinking is pervasive”. J. Scient. Pract. Comput. 1, $67-69$.

Costa, T. et al. (2012) "Trabalhando Fundamentos de Computação no Nível Fundamental: experiência de Licenciandos em Computação da Universidade Federal da Paraíba". XX WEI. Curitiba.

França, R. et al (2012). "Ensino de Ciência da Computação na Educação Básica: Experiências, Desafios e Possibilidades". In: XX WEI Curitiba.

Gros, B. (2007) "The impact of digital games in education". First Monday, v.8, n.7. Disponível em: http://www.firstmonday.org/issues/issue8_7/xyzgros/index.html. Acesso em: 16/12/2014.

Kirkpatrick, D. (1994) "Evaluating Training Programs - The Four Levels". BerrettKoehler Publishers, Inc.

Lima, R. (2006) "Como a relação entre clientes e fornecedores internos à organização pode contribuir para a garantia da qualidade: o caso de uma empresa automobilística". Ouro Preto: UFOP.

Moran, J. (2003). "Mudar a forma de ensinar e aprender com tecnologias: transformar as aulas em pesquisa e comunicação presencial-virtual". Disponível em: http://www.irrodl.org/content/v2.1/downes.pdf. Acesso em junho de 2007.

Nunes, D. (2011). "Ciência da Computação na Educação Básica". Disponível em <http://www.jornaldaciencia.org.br/Detalhe.jsp?id=79207> Acesso em: 25 de out. 2011.

Patton, M. (2002) "Qualitative Research \& Evaluation Methods". 3. ed. California: Sage Publications.

Prieto, L . et al. (2005). "Uso das Tecnologias Digitais em Atividades Didáticas nas Séries Iniciais". Porto Alegre, v. 3, n. 1, p.1-11.

Sá, E. et al. (2007) "Design de atividades de aprendizagem que usam Jogos como princípio para Cooperação". In: Anais do XVIII (SBIE), SP, Brasil.

Scaico, P. D. et al. (2012). Um Relato de Experiências de Estagiários da Licenciatura em Computação com o Ensino de Computação para Crianças. Revista Novas Tecnologias - RENOTE, v.10, n.3.

Shneiderman, B. (2006) “A nova educação: aprendizado eletrônico”. In: SHNEIRDERMAN, B. O laptop de Leonardo: como o novo renascimento já está mudando a sua vida. Rio de Janeiro: Nova Fronteira. p. 129-151.

Wing, J. (2008). "Computational thinking and thinking about computing”. Phil. Trans. R. Soc. A, 366(1881):3717-3725.

Yin, R. (2001) "Estudo de caso - planejamento e métodos". 2. ed. Porto Alegre: Bookman. 